\title{
Uso de wood frame na construção civil no Brasil
}

\author{
Use of the wood frame in the civil construction in Brazil \\ Uso de wood frame in la construccion civil en Brasil
}

Recebido: 06/05/2021 | Revisado: 12/05/2021 | Aceito: 14/05/2021 | Publicado: 30/05/2021

\author{
Evelyn Bastos Resende \\ ORCID: https://orcid.org/0000-0003-4944-5820 \\ Universidade Federal de Alfenas, Brasil \\ E-mail: evelyn.resende@sou.unifal-mg.edu.br \\ Letícia Carolaine Silva Faria \\ ORCID: https://orcid.org/0000-0001-7920-0969 \\ Universidade Federal de Alfenas, Brasil \\ E-mail: leticia.faria@sou.unifal-mg.edu.br \\ Emmanuel Freitas-Ferreira \\ ORCID: https//orcid.org/0000-0002-9399-9380 \\ Universidade Federal de Alfenas, Brasil \\ E-mail: emmanuel.ferreira@ sou.unifal-mg.edu.br \\ Tales Alexandre Aversi-Ferreira \\ ORCID: https://orcid.org/0000-0002-4417-7209 \\ Universidade Federal de Alfenas, Brasil \\ E-mail: tales.ferreira@unifal-mg.edu.br
}

\begin{abstract}
Resumo
O sistema construtivo em wood frame no Brasil vem ganhando espaço pela facilidade de construção, a rapidez na mão de obra, resistência ao suportar as intempéries ambientais de cada região, a competitividade no mercado da construção civil e o principal para as questões sustentáveis. No entanto, a resistência para uso desse tipo de construção no Brasil é alta. O objetivo deste trabalho é apresentar as vantagens e desvantagens das características do sistema construtivo em casas de madeira, abordando as principais responsabilidades sustentáveis e as técnicas usadas. Para a execução deste trabalho foi utilizada como metodologia a pesquisa bibliográfica sistemática. Poucos textos foram encontrados sobre o uso de woodframe no Brasil que mostraram que esse tipo de construção é escasso no país devido a problemas do tipo falta de informação ao publico e aos engenheiros civis nas universidades, falta de mão de oba especializada e problemas de conscientização da população que pensa que esse é um sistema frágil e pouco durável. A melhoria do ensino sobre drywall, logo, também sobre woodframe, deveria ser implantado nas universidades, o engenheiro deveria ser o incentivador e informador sobre as novas técnicas de construção, pelo menos no Brasil.
\end{abstract}

Palavras-chave: Construção civil; Sistema construtivo em wood frame; Sustentabilidade; Responsabilidade social.

\begin{abstract}
The wood frame construction system in Brazil has been increasing due to the spped and ease construction, resistance the environmental weather, the competitiveness in the civil construction and the sustainable issues. However, resistence to use this system of construction in Brazil is high. The objective of this work is to present the advantages and disadvantages of the characteristics of the construction system in woodframe addressing the sustainable responsibilities and the techniques used. For the execution of this work, systematic bibliographic research was performed as methodology. Few texts were found about the use of woodframe in Brazil that showed that this type of construction is scarce in the country due to problems such as lack of information to the public and for civil engineers in universities, lack of skilled workers and problems of awareness of the population that thinks this is a fragile and barely durable system. The improvement of teaching on drywall, therefore, also on woodframe, should be implemented in universities, the engineer should be the encourager and informer about new construction techniques, at least in Brazil.
\end{abstract}

Keywords: Construction; Construction system in wood frame; Sustainability; Social responsability.

\section{Resumen}

El sistema de construcción con woodframe en Brasil ha ido en aumento debido a la rapidez y facilidad de construcción, la resistencia al clima, la competitividad en la construcción civil y los temas de sustentabilidad. Sin embargo, la resistencia para utilizar este sistema de construcción en Brasil es alta. El objetivo de este trabajo es presentar las ventajas y desventajas de las características del sistema constructivo en estructura de madera abordando las responsabilidades sostenibles y las técnicas empleadas. Para la ejecución de este trabajo se realizó como metodología la investigación bibliográfica sistemática. Se encontraron pocos textos sobre el uso de la estructura de 
madera en Brasil que muestren que este tipo de construcción es escasa en el país debido a problemas como la falta de información al público y para los ingenieros civiles en las universidades, la falta de trabajadores calificados y los problemas de consciência de la población que piensa que este es un sistema frágil y pequeño duradero. El perfeccionamiento de la enseñanza en drywall, por lo tanto, también en woodframe, debe ser implementado en las universidades, el ingeniero debe ser el impulsor e informador sobre las nuevas técnicas de construcción, al menos en Brasil.

Palabras clave: Construcción; Sistema constructivo en estructura de madera; Sustentabilidad; Responsabilidad social.

\section{Introdução}

Apesar da Construção Civil passar por constantes inovações tecnológicas, o Brasil adota uma postura conservadora ao utilizar majoritariamente estruturas convencionais de alvenaria ao menos para as construções de pequeno e médio porte (Gomes, et al., 2013; Labuto, 2014) gerando desperdício de materiais (Vieira, 2006; Costa \& Nascimento, 2015). A construção em alvenaria é considerada como um sistema ultrapassado e, em muitos países, é substituído por drywall como no Japão, Europa, Estados Unidos (Ribaski, Dudek \& Rotta, 2017), Canadá (Nunes, 2015).

A tecnologia drywall ainda é recente no Brasil o que pode significar uma oportunidade de exploração desse tipo de construção como alternativa para novas estratégias econômicas e com grande potencial competitivo para a indústria madeireira nacional (Ribaski, Dudek \& Rotta, 2017); não obstante, a construção civil usa a madeira serrada e beneficiada como tábuas, vigas, caibros, pranchas, pontaletes e ripas, gesso e o incipientemente o steelframe (Pfeil \& Pfeil, 2003; Aversi-Ferreira, 2018).

Em específico para o uso da madeira na construção civil como material principal na estrutura de casas e edifícios, o atraso brasileiro torna-se mais evidente quando comparado aos Estado Unidos, cujas tecnologias com uso da madeira foram inventadas logo após a colonização europeia, por volta de 1833, e representa $95 \%$ das casas construídas atualmente (Zaparte, 2014).

De fato, a madeira pode ser considerada um dos mais antigos materiais de construção devido a sua disponibilidade na natureza além de poder ser manipulada relativamente rápida e facilmente; era usada para construir casas e pontes, abrir estradas (Bittencour \& Oliveira, 2009) apresenta uma relação resistência/peso alta em comparação a outros materiais (Pfeil \& Pfeil, 2003; Molina \& Calil, 2010).

De um modo geral, contrariando o senso comum, construções em madeira utilizam material sustentável, renovável pois todas são oriundas do reflorestamento, sem produção de poluentes e com baixo custo energético (Junior \& Molina, 2010).

Considerando as características de sustentabilidade, os valores estéticos e tornando as habitações mais aconchegantes, a madeira é um material que deveria ser mais usado na construção civil. Em consequência, este artigo tem como objetivo analisar as construções em wood frame com suas vantagens e desvantagens, citar as técnicas usadas nesse tipo de construção e relacionar as principais responsabilidades em termos de sustentabilidade associadas ao wood frame. Para tal, foi realizada uma revisão sistemática da literatura para mostrar a viabilidade do uso da madeira na construção civil.

Deve-se considerar a escassez de artigos científicos sobre o wood frame no Brasil, considerando ser um tema inovador para a engenharia atual, arcaica comparada com outros países e desvalorizada por uma cultura onde não se valoriza o meio ambiente e maneiras de não sobrecarregá-lo.

\section{Metodologia}

Foi realizada uma pesquisa bibliográfica sistemática em busca de dados sobre o uso da madeira nas construções brasileiras, o seu impacto ambiental e a sustentabilidade. As buscas pelos descritores foram as palavras chaves dos periódicos, como engenharia civil e madeira, engenharia civil e wood frame, engenharia civil e materiais sustentáveis, engenharia civil e sustentabilidade, buscados na plataforma Capes que envolvem Scielo, Elsevier, Springer somado ao Google acadêmico. Foram considerados os textos em português como os principais e alguns em inglês para fundamentar as bases do trabalho. Foi 
considerada também a relevância do periódico em relação ao seu fator de impacto, priorizando o alcance de leitores. Livros considerados clássicos como Estruturas de Madeira (Pfeil \& Pfeil, 2003) e também normas técnicas, dissertações/teses foram usadas (Figura 1). Na figura 2 os dados sobre engenharia e sustentabilidade foram quantificados.

Os critérios de exclusão foram artigos estrangeiros que não versavam sobre o uso de wood frame no Brasil. Devido à escassez de artigos e textos, a data mínima não foi considerada.

\section{Resultados e Discussão}

$\mathrm{Na}$ busca de dados foi encontrada uma escassa quantidade de artigos científicos sobre wood frame, o que representa a falta de informações sobre esse tipo de construção civil no Brasil, que além de insuficiente em números de execução, também sofre na deficiência de trabalhos acadêmicos. Artigos sobre sustentabilidade são vários no Brasil e no mundo, mas relacionados com wood frame são também escassos, então, para fundamentar as bases do artigo foram usados, além dos artigos, livros e teses/dissertações (tabela 1)

Para sumarizar, os artigos estudados foram divididos em dois grupos, os de engenharia que envolvem madeira e wood frame e sustentabilidade, que engloba matérias sustentáveis (Tabela 1, Figura 2).

Tabela 1. Dados dos artigos usados neste trabalho identificando os títulos, assuntos e tipo de texto.

\begin{tabular}{|c|c|c|c|}
\hline & Artigos e livros & Assunto & $\begin{array}{l}\text { Tipo de } \\
\text { texto }\end{array}$ \\
\hline 1. & $\begin{array}{l}\text { Associação Brasileira de normas técnicas (1997). NBR-7190 - Projeto de estruturas de } \\
\text { madeira: procedimento. Rio de Janeiro: ABNT. }\end{array}$ & Engenharia & Norma \\
\hline 2. & $\begin{array}{l}\text { Aversi-Ferreira, T. A. (2018). Alguns comentários sobre o uso das construções do tipo dry wall } \\
\text { no Brasil. Engineering Sciences, } 6(1), 21-31 \text {. }\end{array}$ & Engenharia & Artigo \\
\hline 3. & $\begin{array}{l}\text { Barbisan, A. O.; Spadotto. A.; Dalla N. D.; Lopes, T. E. C \& Wergenes, T. N. (2012). Impactos } \\
\text { ambientais causados pela construção civil. Unoesc \& Ciência-ACSA, 2(2), 173-180. }\end{array}$ & Sustentabilidade & Artigo \\
\hline 4. & $\begin{array}{l}\text { Bissoli-Dalvi, M.; Ferres, C. S.; Alvarez, C. E. \& Fuica, G. E. S. (2017). Avaliação da } \\
\text { Sustentabilidade da Madeira por Meio da Ferramenta. ISMAS, 24(1), } 8087 .\end{array}$ & Sustentabilidade & Artigo \\
\hline 5. & $\begin{array}{l}\text { Bittencourt, L. P.; Oliveira, G. B. (2009). A indústria madeireira paranaense nos anos recentes. } \\
\text { Revista das Faculdades Santa Cruz. 7(1), 33-42. }\end{array}$ & Engenharia & Artigo \\
\hline 6. & $\begin{array}{l}\text { Calil Junior, C \& Molina, J. C. (2010). Cobertura em estruturas de madeira: exemplos de } \\
\text { cálculo. Editora, Pini, São Paulo. }\end{array}$ & Engenharia & Livro \\
\hline 7. & $\begin{array}{l}\text { Calil Junior, C.; Lahr, F. A. R. \& Dias, A. A. (2003). Dimensionamento de elementos } \\
\text { estruturais de madeira, Livraria Online e Editora Manole, Barueri. }\end{array}$ & Engenharia & Livro \\
\hline 8. & $\begin{array}{l}\text { Corrêa, R. L. (2009). Monografia: sustentabilidade na construção civil, UFMG, Belo } \\
\text { Horizonte. }\end{array}$ & Sustentabilidade & Monografia \\
\hline 9. & $\begin{array}{l}\text { Chau, K. W. (2007). Incorporation of Sustainability Concepts into a Civil Engineering } \\
\text { Curriculum. Journal of Professional Issues in Engineering Education and Practice, 133(3), } \\
\text { 188-191. doi: https://doi.org/10.1061/(ASCE)1052-3928(2007)133:3(188) }\end{array}$ & Sustentabilidade & Artigo \\
\hline
\end{tabular}




\begin{tabular}{|c|c|c|c|}
\hline 10. & $\begin{array}{l}\text { Costa, A. T \& Nascimento, F. B. C. (2015). Uso de gesso acartonado em vedações internas. } \\
\text { Cadernos de graduação. 2(3), 99-106. }\end{array}$ & Engenharia & Artigo \\
\hline 11. & $\begin{array}{l}\text { Espindola, R. L. (2014). Inserção e financiamento do sistema Wood Frame no programa } \\
\text { habitacional Minha Casa Minha Vida, 1578-1587. }\end{array}$ & Engenharia & Artigo \\
\hline 12. & $\begin{array}{l}\text { Gasques, A. C.; Okawa, C.; M, Angelis Neto, G.; Miotto, J. L. \& Castro, T. R. (2014). } \\
\text { Impactos ambientais dos materiais da construção civil: breve revisão teórica, 23, 13-24. }\end{array}$ & Sustentabilidade & Artigo \\
\hline 13. & $\begin{array}{l}\text { Gomes, C. E. M.; Vivian, A. L.; Sichieri, E. P. \& Paliari, J. C. (2013). Light steel frame na } \\
\text { produção de moradias no Brasil, IX Congresso de Construção Metálica e Mista \& I Congresso } \\
\text { Luso-Brasileiro de Construção Metálica Sustentável. Porto. }\end{array}$ & Engenharia & Artigo \\
\hline 14. & $\begin{array}{l}\text { Labuto, L. V. (2014). Parede seca - sistema construtivo de fechamento de estrutura de drywall. } \\
\text { Trabalho de Conclusão de Curso, Universidade Federal de Minas Gerais. Belo Horizonte. }\end{array}$ & Engenharia & Monografia \\
\hline 15. & $\begin{array}{l}\text { Lacerda, J. F. S. B. \& Gomes, J. de O. (2014). Uma visão mais sustentável dos sistemas } \\
\text { construtivos no Brasil: análise do estado da arte. Revista E-Tech: Tecnologias Para } \\
\text { Competitividade Industrial. 7(2), 167-186. https://doi.org/10.18624/e-tech.v7i2.469 }\end{array}$ & Sustentabilidade & Artigo \\
\hline 16. & $\begin{array}{l}\text { López, H. H.; Escamilla, A. C. \& Pérez, I. M. (2019). Life Cycle Assessment of dwellings: a } \\
\text { case of study that contrasts masonry wall with wood-frame wall in a social housing. Anales de } \\
\text { Edificación, 5(3), 76-91. }\end{array}$ & Engenharia & Artigo \\
\hline 17. & $\begin{array}{l}\text { Marques, M. E. L. (2008). O papel da madeira na sustentabilidade da construção, Dissertação } \\
\text { submetida para satisfação parcial dos requisitos do grau de mestre em engenharia.Portugal. }\end{array}$ & Sustentabilidade & Dissertação \\
\hline 18. & $\begin{array}{l}\text { Mauro, D. \& Fabio, J. P. (2013). Madeira na construção civil: da ilegalidade à certificação, } \\
\text { Dissertação, Universidade Estadual de Campinas, Campinas. }\end{array}$ & Engenharia & Dissertação \\
\hline 19. & $\begin{array}{l}\text { Molina, J. C \& Calil, J. C (2010). Sistema construtivo em wood frame para casas de madeira, } \\
\text { Semina: Ciências Exatas e Tecnológicas, Londrina, 31(2), 143-156. }\end{array}$ & Engenharia & Artigo \\
\hline 20. & $\begin{array}{l}\text { Ng, T. S.; Chan, C. K. G.; Chan, L. H. J. \& Zou, W. W. J. (2016). Environmental impacts of } \\
\text { construction material production, Engineering Sustainability, 170(3), 169-184. }\end{array}$ & Engenharia & Artigo \\
\hline 21. & $\begin{array}{l}\text { Nunes, H. P. (2015). Estudo da aplicação do Drywall em edificação vertical, Trabalho de } \\
\text { conclusão de curso (TCC), Universidade Tecnológica Federal do Paraná. }\end{array}$ & Engenharia & $\begin{array}{l}\text { Trabalho de } \\
\text { conclusão de } \\
\text { curso [TCC] }\end{array}$ \\
\hline 22. & Pfeil, W. \& Pfeil, M. (2013). Estruturas de Madeira. 6ª ed, LTC: Rio de Janeiro. 240. & Engenharia & Livro \\
\hline 23. & $\begin{array}{l}\text { Ribaski, N. G.; Dudek, L. C. \& Rotta, C. E. (2017). O sistema wood frame e o setor madeireiro } \\
\text { paranaense. Brazilian Applied Science Review. 1(1), 34-41. }\end{array}$ & Engenharia & Artigo \\
\hline 24. & $\begin{array}{l}\text { Sandanayake, M.; Zhang, G.; Setunge, S.; Luo, W. \& Chun-Qing, L. (2017). Estimation and } \\
\text { comparison of environmental emissions and impacts at foundation and structure construction } \\
\text { stages of a building - A case study, Journal of Cleaner Production, 319-329. }\end{array}$ & Sustentabilidade & Artigo \\
\hline
\end{tabular}




\begin{tabular}{|l|l|l|l|}
\hline 25. & $\begin{array}{l}\text { Vasques, C. P. F. \& Pisso, L. M. B. (2014). Comparativo de sistemas construtivos, } \\
\text { convencional e wood frame em residências unifamiliares, Cognitio. 1,1-17. }\end{array}$ & Engenharia \\
\hline 26. & $\begin{array}{l}\text { Vieira, H. (2016). Logística aplicada à construção civil como melhorar o fluxo de produção nas } \\
\text { obras, Editora Pini. }\end{array}$ & Engenharia & Livro \\
\hline 27. & $\begin{array}{l}\text { Zaccai, E. (2012). Over two decades in pursuit of sustainable development: Influence, } \\
\text { transformations, limits, Environmental Development, 79-90. }\end{array}$ & Sustentabilidade & Artigo \\
\hline 28. & $\begin{array}{l}\text { Zaparte, T. A. (2014). Estudo e adequação dos principais elementos do modelo canadense de } \\
\text { construção em Wood Frame para o Brasil, TCC - Universidade Tecnológica Federal do Paraná } \\
\text { - UTFPR. }\end{array}$ & Engenharia & TCC \\
\hline 29. & $\begin{array}{l}\text { Zenid, G. J. (2009). Madeira: Uso sustentável na construção civil, Instituto de Pesquisas } \\
\text { Tecnológicas, 2a ed, 99. }\end{array}$ & Engenharia & Livro \\
\hline
\end{tabular}

Fonte: Autores (2021)

Figura 1: Porcentagem de tipos de textos estudados no artigo. Observe que a quantidade de artigos é cerca de $30 \%$ maior que os textos gerais de TCC, dissertações e monografias. Apenas uma norma foi utilizada devido à escassez das mesmas na ABNT.

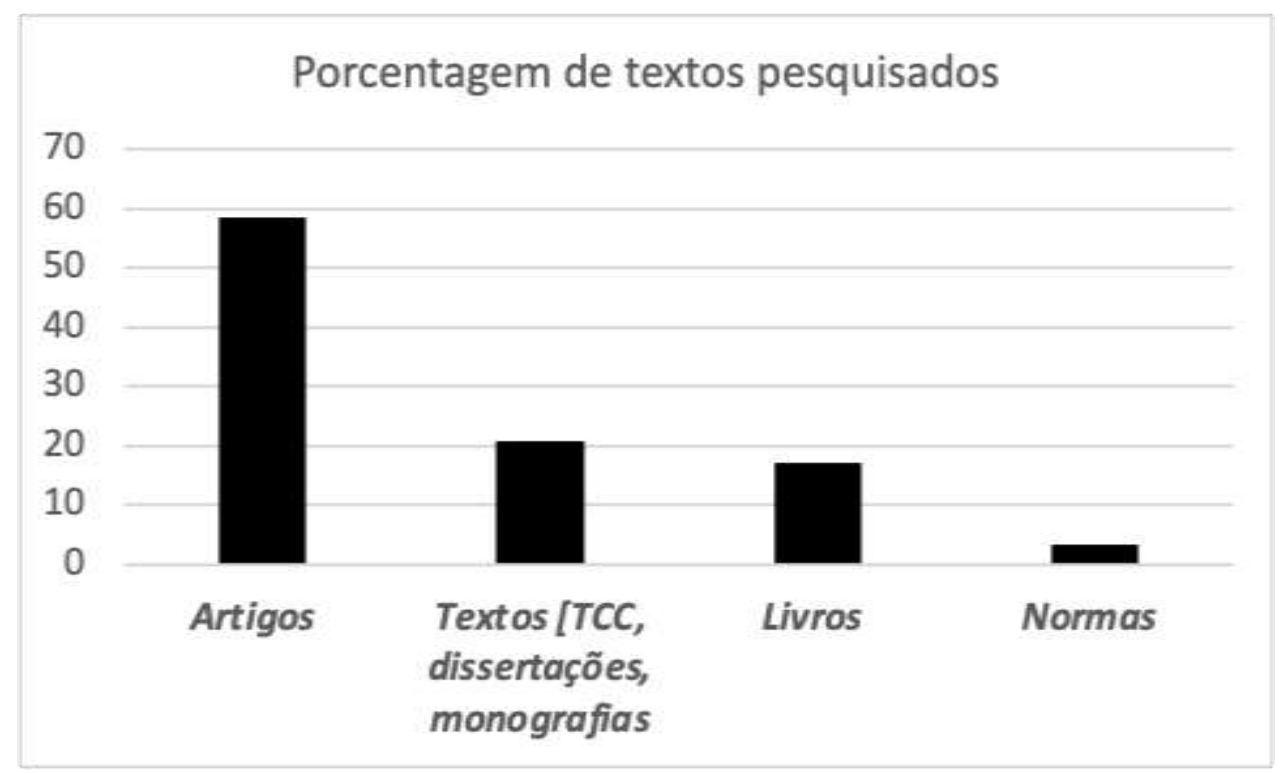

Fonte: Autores (2021). 
Figure 2:Porcentagem dos assuntos básicos Sustentabilidade e Engenharia, estudados no artigo. Os artigos que versam sobre engenharia usados neste artigo foram mais que $50 \%$ a mais que os que versam sobre sustentabilidade.

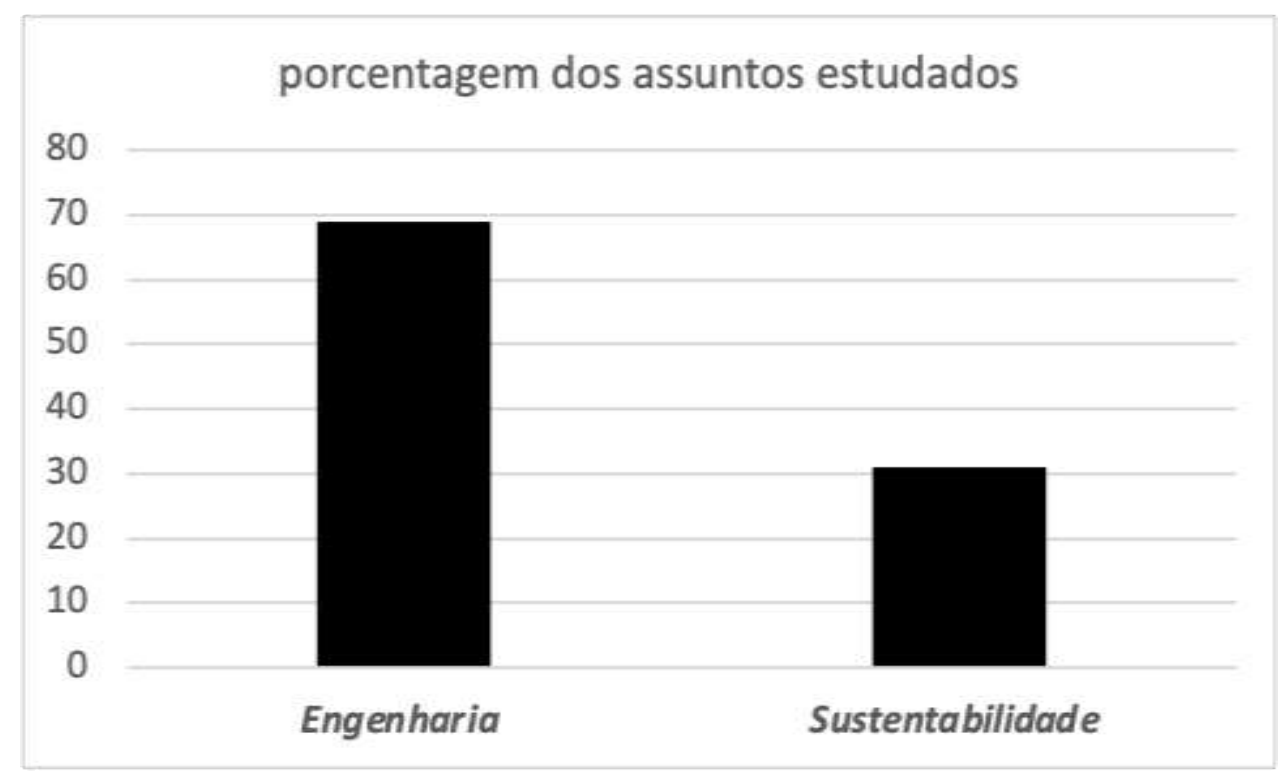

Fonte: Autores (2021).

\subsection{Uso da madeira pela construção civil no Brasil}

Devido a cultura brasileira de que usar madeira na estrutura de casas geraria construções fracas e de rápido desgaste e a de usar alvenaria pela herança portuguesa (uma vez que Portugal não possuía florestas) que é um método artesanal de construção (Lacerda \& Gomes, 2014), a tecnologia de construção wood frame é incipiente no país; mas a madeira é utilizada extensivamente para construção de telhados, pontes, alguns acabamentos e móveis (Mauro \& Fábio, 2013; Pfeil \& Pfeil, 2003).

As características da cultura do Brasil mais a falta de informações sobre as novas tecnologias de construção civil, pode ser uma das causas do atraso do Brasil no uso de tecnologias dry wall, especificamente a wood frame (Aversi-Ferreira, 2018). No Paraná, por exemplo, ainda há o preconceito de que as casas de madeira são para as pessoas de baixa e média renda (Ribaski, Dudek \& Rotta, 2017).

A tecnologia de wood frame somente chegou no país no início dos anos 90 do século passado, mas desde antes e ainda recentemente, o país não possui uma legislação específica para o uso do wood frame na construção civil, levando os engenheiros civis a adotarem a norma europeia (EUROCODE 5) (Pfeil \& Pfeil, 2003; Zaparte, 2014). A norma Brasileira mais adaptada para o uso de madeira como base da construção civil de casas e edifícios é a NBR 7190 (1997) e outra opção é usar livros específicos para o dimensionamento das placas (Junior \& Dias, 2003).

Historicamente a extração da madeira começou de modo devastador no Brasil devido a chegada da família imperial, gerando comprometimento de várias espécies, e ainda nos dias atuais ainda existe a extração ilegal, no entanto, a construção do tipo wood frame tem como base a madeira de reflorestamento, nunca a nativa (Pereira \& Cunha, 2003).

Atualmente, seguindo os modelos internacionais, algumas empresas brasileiras começaram a realizar testes com construções wood frame. Um exemplo é a Construtora Roberto Ferreira, a qual executou juntamente ao grupo i Verde um projeto de 280 habitações em Pelotas, RS, no ano de 2012 (Zaparte, 2014).

Tal projeto permitiu obter dados sobre o uso do wood frame na construção civil nacional e permitiu constatar que, apesar do custo da madeira ser 5\% maior que a alvenaria, essa tecnologia é mais econômica que os métodos tradicionais no pre $\approx$ ço final do edifício por apresentar uma execução mais rápida e a economia ocorre pela menor quantidade de mão de obra utilizada, além da maior racionalização de materiais gerando menos resíduos, aspecto que torna o wood frame mais sustentável 
que a alvenaria; pelo menos na questão do uso da madeira reflorestada que implica a redução do desmatamento de florestas nativas no Brasil (Vasques \& Pisso, 2014; Zaparte, 2014), além de, também, apresenta maior conforto termo-acústico.

Entre as principais desvantagens do uso da tecnologia wood frame no Brasil está o desconhecimento do uso adequado do método, a falta de mão de obra especializada, a altura das edificações serem limitadas ao máximo de 5 pavimentos, a baixa oferta de ferramentas específicas e a resistência do mercado às mudanças (Vasques \& Pisso, 2014).

Os motivos para os escolhos ligados à construção wood frame, inter alia, pode ser a falta de trabalhos acadêmicocientíficos na área (ver Resultados) e o desinteresse dos engenheiros em aplicarem métodos diferentes do tradicional nas construções, podendo-se somar a falta de disciplinas voltadas para construções em wood frame nas faculdades de engenharia (Aversi-Ferreira, 2018; Molina \& Junior, 2010; Zaparte, 2014).

De um modo mais geral, considerando que o Brasil apresenta um déficit residencial (número de residências) para a população, a situação descrita acima deve ser mudada, especialmente considerando que a tecnologia wood frame utilizada nos países desenvolvidos torna as construções mais confortáveis e acessíveis, devido a velocidade na construção, economia e conforto oferecidos (Gomes, et al, 2013; Labuto, 2014; Vasques \& Pisso, 2014; Zaparte, 2014).

No Brasil a madeira ainda é empregada na construção civil como ferramenta na execução da obra, nos andaimes, escoramentos, instalação do canteiro e nas formas para colunas com estrutura temporária. A utilização da madeira como definitiva nas obras de alvenaria são as coberturas para telhados, esquadrias, forros e pisos. São geralmente utilizadas em estruturas de classe alta pelo fato de ser um material mais caro no mercado, por ser madeira mais resistente e para a classe alta (Zenid, 2009).

Apesar dos problemas citados, o uso da madeira na construção civil com o intuito de economizar e gerar um desenvolvimento sustentável tem aumentado a preocupação com a exploração da madeira. Segundo Zenid (2009) estudos mostram que se for escolhido um critério para seguir durante as etapas do projeto de construção pode ser vantajoso usando as premissas de escolhas de materiais sustentáveis, como recursos renováveis que podem sempre estar na lista de prioridades de uma execução de obra, que é uma responsabilidade do engenheiro que precisa informar os clientes sobre isso. Nesse sentido o wood frame se encaixa como uma das opções além das tecnologias dry wall em geral.

Nesse caminho é necessário criar uma ferramenta que auxilie o projetista na etapa de escolha de materiais que gerencie a economia de materiais e a gestão de resíduos (Bissoli-Dalvi, et al., 2017). Das várias ferramentas, o autor citado recomenda a ISMAS (Seleção de Materiais Mais Sustentáveis), tendo como principal característica o uso simples, rápido e prático. O uso da ISMAS ocorre quando se quer adotar critérios ambientais de sustentabilidade que são medidos em pesos os critérios usados para a obtenção da sustentabilidade na obra, que são sete: 1] reaproveitado no todo ou parte; 2] é renovável; 3] dispensa materiais adicionais para acabamento; 4] possui elementos reciclados; 5] a durabilidade independe de manutenção; 6] favorece a desmontagem visando o reaproveitamento e; 7] favorece a baixa geração de resíduos. Esse é o procedimento permite obter uma escolha de materiais renováveis para o projeto de forma mais sustentável.

\subsection{Aspectos sobre construção em wood frame}

O sistema de construção wood frame no Brasil iniciou no ano de 2001 quando foi construída a primeira casa de madeira nesse sistema na cidade de Viamão no Rio Grande do Sul, pelo engenheiro Carlos Alves. Esta tecnologia foi inventada pelos Alemães possibilitando a construção de casas com 200 metros quadrados em 60 dias de trabalho economizando mão de obra e matéria prima (Molina \& Junior, 2010).

Em termos gerais e para exemplificar como poderia ser usado o sistema de construção wood frame no Brasil, as etapas e montagens na construção são: 1] fundação da estrutura que é escolhida juntamente com o projeto e o tipo de solo de cada lugar; 2] pisos que são feitas por chapas e compensados de madeira e ou chapas cimentícias; 3] paredes são compostas 
por painéis, montantes verticais e ferros galvanizados para a estrutura das parede entre as chapas; 4] sistema elétrico e hidráulico são idênticos ao sistema de uma casa de alvenaria, elas são embutidas nas chapas das paredes de madeira; 5] revestimentos são feitos por madeira, PVC e aço, podendo também ser revestidos por placas cimentícias como o acabamento de alvenaria; 6] contraventamento é feito com a fixação de placas nas partes externas das paredes e telhado em treliças de madeira e com conectores de chapas de dentes estampados.

Esse sistema permite maior controle de qualidade com redução acentuada de resíduos e diminuição em até 3 vezes o tempo de construção (Ribaski, Dudek \& Rotta, 2017), por exemplo, em alguns países como na Estados Unidos, Alemanha, Chile, é construída toda a estrutura da casa na indústria e depois ela é transportada para o local definitivo (Molina \& Junior, 2010). A madeira usada para essas construções são o pinus e/ou eucalipto, neste contexto o pinus se destaca por ser uma madeira mais leve e de fácil tratamento.

O eucalipto é comercialmente utilizado para a construção de casas de madeira pela facilidade de desdobramento da madeira e as matas de reflorestamento que podem ser encontradas em todo o Brasil, sendo uma opção viável e sustentável, diminuindo a pegada do carbono com relação às madeiras que eram serradas das florestas nativas da Amazônia para a comercialização nos grandes centros do país, apesar dessas madeiras não poderem, atualmente, serem usadas no método wood frame.

Somado ao problema do uso de madeiras não reflorestadas é que no transporte das madeiras derivadas de mata nativa a emissão de $\mathrm{CO}_{2}$ através da queima de combustíveis fósseis é alta (Sandanayake, 2017).

Devido ao exposto, percebe-se que o Brasil é um país que pode ter um grande potencial para produzir casas feitas de madeira, pelo menos pela quantidade e disponibilidade de áreas de reflorestamento. No sul do país existem empresas que prestam este serviço, mas é pouco usado em outras regiões, pode ser pelo fato de costumes e tradições que já está enraizada na cultura brasileira no uso alvenaria ou por questões financeiras. Um caminho para sanar esses problemas de falta de construções usando recursos sustentáveis é aperfeiçoar os profissionais na área de engenharia e arquitetura, somado à adição de disciplinas que contemplem as construções diferentes de alvenaria nas universidades (Aversi-Ferreira, 2018; Molina \& Junior, 2010).

De fato, há mais economia, pelo menos teoricamente, em usar o sistema wood frame no Brasil, pois este recebe apoio financeiro de bancos como a Caixa Econômica Federal (Ribaski, Dudek \& Rotta, 2017), que tem priorizado o financiamento para construções em wood frame comparado com as construções convencionais, facilitando a construção da idealizada casa própria com recursos sustentáveis (Molina \& Junior, 2010), outros bancos como o Banco do Brasil e bancos credenciados no programa "Minha Casa Minha Vida (MCMV)" fornecem financiamento para os sistemas construtivos do tipo dry wall (Lacerda \& Gomes, 2014); por exemplo, através do programa MCMV) em 2012, foi executado o primeiro conjunto habitacional em wood frame no Brasil (Espíndola, 2014).

\subsection{Sustentabilidade e uso da madeira na construção civil.}

Os principais debates sobre sustentabilidade foram iniciados na década de 80 com o relatório Brundtland que é um documento intitulado Nosso Futuro Comum (Our Common Future), publicado em 1987. Coordenado pela então primeiraministra da Noruega, Gro Harlem Brundtland, a Comissão Mundial sobre Meio Ambiente e Desenvolvimento originou um documento no qual houve a disseminação da ideia de desenvolvimento sustentável (Corrêa, 2009).

O conceito vinha sendo concebido desde a década de 1970, pois problemas relacionados ao meio ambiente já eram graves e havia necessidade um órgão responsável para administrar a situação que era: suprir as necessidades da geração presente sem afetar a habilidade das gerações futuras de suprir as suas (Corrêa, 2009). Em seguida, houve a Conferência das Nações Unidas sobre o Meio Ambiente e Desenvolvimento (UNCED) em 1992 que foi fomentou uma declaração Brasileira sobre estas questões. Desde então, pesquisas em todo o mundo sobre engenharia levaram em consideração aspectos sobre 
sustentabilidade na construção civil (Ng, et al., 2016; Zaccai, 2012) em acordo com as propostas modernas de criar um futuro melhor para as gerações posteriores.

A sustentabilidade deve ser um fator de interesse e efetivação em todas as áreas, com suas múltiplas implicações e precisa ser executada em todos os parâmetros da construção civil que abrange, pois abrange toda a sociedade (Chau, 2007), e, considerando pelo menos 3 aspectos: o econômico, o social e o ambiental (Lacerda \& Gomes, 2014). Para viabilizar o compromisso das empresas da cadeia produtiva com o processo renovável, é preciso criar bases para projetos com materiais sustentáveis e ser interessante economicamente com um exequível custo-benefício, busca constante pela qualidade, devendo ser sustentável no seu processo operacional (Corrêa, 2009).

A construção em alvenaria no Brasil é feita via um sistema artesanal, pouco produtivo e com geração de grande quantidade de resíduos em contraposição às considerações sustentáveis, apresentando um caráter não homogêneo e não seriado de produção, dependente de fatores climáticos para realização do processo construtivo, da contratação de várias empresas que não trabalham em conjunto na maioria das vezes e com alta rotatividade de empregados (Lacerda \& Gomes, 2014).

Por outro lado, de acordo com a Agenda 21 para a construção sustentável (que foi um dos principais resultados da conferência Rio-92), durante as fases primárias das construções são consumidos $50 \%$ dos recursos naturais e produzidos $50 \%$ de resíduos a menos nas construções do tipo sustentável em relação à alvenaria (Marques, 2008).

Para minorar esse problema, a utilização de materiais eco-eficientes é um bom ponto de partida para a construção sustentável, que não são nocivos à camada de ozônio, são duráveis, exigem poucas operações de manutenção, devem estar disponíveis nas proximidades dos locais de construção, serem elaborado a partir de matérias recicladas e/ou que possuam grandes potencialidades para virem a ser recicladas ou reutilizadas. Padrões estes em que a madeira reflorestada se encaixa eficientemente como matéria prima sustentável para este modelo de construção, portanto, mostrando as vantagens sustentáveis do wood frame. A madeira é o único material de construção (relativamente aos principais como aço, alumínio entre outros) que é reciclável, renovável e biodegradável (Lacerda \& Gomes, 2014) e o produto que tem o menor gasto de energia para sua transformação (Marques, 2008).

A sustentabilidade da madeira no setor construtivo está respaldada pelas certificações que impulsionam as empresas a introduzirem em seus processos produtivos as questões ambientais e sociais (Ribaski, Dudek \& Rotta, 2017). De fato, a certificação florestal e ambiental no Brasil tem alguns programas específicos como a Cerflor (Programa Brasileiro de Certificação Florestal), AQUA (Certificação Internacional de Qualidade Ambiental de Edificações) e FSC (Forest Stewardship Council), que caucionam a extração da madeira de forma correta em manejo e para atenuar os impactos negativos nas florestas controlando a redução da emissão de $\mathrm{CO}_{2}$.

Uma construção à base de madeira, além de beneficiar com um maior conforto térmico e acústico, possui inúmeros benefícios para a sociedade, por exemplo, em regiões frias o conforto térmico é maior do que a alvenaria convencional, e um estudo recente feito em casas no Chile avaliando o ciclo de vida de moradias, afirma que paredes feita de madeira gera cerca de $1 / 3$ a menos de economia térmica em relação às de alvenaria (López, et al., 2019).

Uma parte essencial do desenvolvimento sustentável é a resiliência a desastres naturais das residências de wood frame, estes desastres são inevitáveis, mas é possível criar condições para que quando ocorram, seja mínima a perda, como ocorre no Japão. Testes feitos com casas de madeira simples e residência com estrutura de madeira tradicional têm boa capacidade de suportar sismos, as juntas de estruturas de madeira podem dissipar bem a energia em um terremoto, portanto protegendo a estrutura principal de danos significativos e do colapso. A facilidade de construção/reconstrução de casas de madeira e a diminuição do peso dos escombros também podem ser vistas como uma vantagem estrutural do uso de wood frame (Barbisan, et al., 2019). 


\section{Conclusão}

Este artigo apresentou uma revisão da literatura engajada em sistema construtivo de madeira do tipo wood frame considerando aspectos de sustentabilidade, com o objetivo de relatar os impactos ambientais causados pela geração de resíduos durante o processo construtivo em uma obra de alvenaria como o desperdício de água, o aumento do uso de energia e o armazenamento incorreto de materiais que pode poluir o solo, a água e o ar, tudo isso minimizado ou evitado quando se usa o wood frame.

As vantagens da construção com o sistema wood frame com relação ao de alvenaria são a rápida entrega da obra, o uso de materiais sustentáveis e renováveis, maior conforto térmico, menor custo total do edifício. A desvantagem atualmente no Brasil é que o sistema é uma nova tecnologia, portanto, com falta de mão de obra especializada, pouco conhecimento da técnica de engenheiros e arquitetos, falta de disciplinas especificas sobre dry wall e construções sustentáveis nas faculdades de engenharia/arquitetura, cultura arraigada em construções de alvenaria, preconceito em relação ao status social de quem mofra em casa de madeira.

Incentivos nos cursos de graduação no assunto de sistema construtivos de madeira ou sobre a sustentabilidade juntamente com a construção mais limpa, assim formando engenheiros mais conscientes na sociedade, dando ênfase nas construções sustentáveis, são caminhos que facilitarão a disseminação das vantagens do uso de wood frame como via de construção civil no país.

É forçoso, e acordo com os dados desse artigo, que mais estudos sobre a necessidade e importância do uso do tipo de construção wood frame no Brasil, de um lado por motivos acadêmicos, e, pelo outro, de gerar maior sustentabilidade na construção civil para o país não se manter tão atrasado nas técnicas de construção em relação aos países desenvolvidos.

\section{Agradecimentos}

Os autores agradecem aos prestimosos comentários feitos pelos revisores anônimos que melhoraram a apresentação e qualidade cientifica desse artigo.

\section{Referências}

Associação Brasileira de normas técnicas (1997). NBR-7190 - Projeto de estruturas de madeira: procedimento. ABNT.

Aversi-Ferreira, T. A. (2018). Alguns comentários sobre o uso das construções do tipo dry wall no Brasil. Engineering Sciences, 6(1), 21-31. 10.6008/CBPC2318-3055.2018.001.0003

Barbisan, A. O., Spadotto. A., Dalla N. D., Lopes, T. E. C \& Wergenes, T. N. (2012). Impactos ambientais causados pela construção civil. Unoesc \& Ciência ACSA, 2(2), 173-180.

Bissoli-Dalvi, M., Ferres, C. S., Alvarez, C. E. \& Fuica, G. E. S. (2017). Avaliação da Sustentabilidade da Madeira por Meio da Ferramenta. ISMAS, 24(1), 8087. https://doi.org/10.1590/2179-8087.077214

Bittencourt, L. P., Oliveira, G. B. (2009). A indústria madeireira paranaense nos anos recentes. Revista das Faculdades Santa Cruz, 7(1), 33-42.

Calil Junior, C., Lahr, F. A. R. \& Dias, A. A. (2003). Dimensionamento de elementos estruturais de madeira. Barueri, Livraria Online e Editora Manole.

Calil Junior, C \& Molina, J. C. (2010). Cobertura em estruturas de madeira: exemplos de cálculo. Editora, Pini.

Chau, K. W. (2007). Incorporation of Sustainability Concepts into a Civil Engineering Curriculum. Journal of Professional Issues in Engineering Education and Practice, 133(3), 188-191. https://doi.org/10.1061/(ASCE)1052-3928(2007)133:3(188)

Costa, A. T \& Nascimento, F. B. C. (2015). Uso de gesso acartonado em vedações internas. Cadernos de graduação, 2(3), 99-106.

Corrêa, R. L. (2009). Monografia: sustentabilidade na construção civil, UFMG, Belo Horizonte.

Espindola, R. L. (2014). Inserção e financiamento do sistema Wood Frame no programa habitacional Minha Casa Minha Vida. In XV Encontro Nacional de Tecnologia do Ambiente Construído, Maceió, 1578-1587. 10.17012/entac2014.566. 
Research, Society and Development, v. 10, n. 6, e31210615818, 2021

(CC BY 4.0) | ISSN 2525-3409 | DOI: http://dx.doi.org/10.33448/rsd-v10i6.15818

Gasques, A. C. (2015). Impactos ambientais dos materiais da construção civil: Breve revisão teórica. Revista Tecnológica, 23(1), 13-24. https://doi.org/10.4025/revtecnol.v23i1.23375

Gomes, C. E. M., Vivian, A. L., Sichieri, E. P. \& Paliari, J. C. (2013). Light steel frame na produção de moradias no Brasil, In IX Congresso de Construção Metálica e Mista \& I Congresso Luso-Brasileiro de Construção Metálica Sustentável. Porto.

Labuto, L. V. (2014). Parede seca - sistema construtivo de fechamento de estrutura de drywall. Trabalho de Conclusão de Curso, Universidade Federal de Minas Gerais. Belo Horizonte.

Lacerda, J. F. S. B. \& Gomes, J. de O. (2014). Uma visão mais sustentável dos sistemas construtivos no Brasil: análise do estado da arte. Revista E-Tech: Tecnologias Para Competitividade Industrial, 7(2), 167-186. https://doi.org/10.18624/e-tech.v7i2.469

López, H. H., Escamilla, A. C. \& Pérez, I. M. (2019). Life Cycle Assessment of dwellings: a case of study that contrasts masonry wall with wood-frame wall in a social housing. Anales de Edificación, 5(3), 76-91. 10.20868/ade.2019.4374.

Marques, M. E. L. (2008). O papel da madeira na sustentabilidade da construção. Dissertação, Universidade do Porto, Cidade do Porto.

Mauro, D. \& Fabio, J. P. (2013). Madeira na construção civil: da ilegalidade à certificação. Dissertação, Universidade Estadual de Campinas, Campinas.

Molina, J. C \& Calil, J. C (2010). Sistema construtivo em wood frame para casas de madeira. Semina: Ciências Exatas e Tecnológicas, 31(2), 143-156. http://dx.doi.org/10.5433/1679-0375.2010v31n2p143.

Ng, T. S., Chan, C. K. G., Chan, L. H. J. \& Zou, W. W. J. (2016). Environmental impacts of construction material production. Engineering Sustainability, 170(3), 169-184. 10.1680/jensu.15.00009.

Nunes, H. P. (2015). Estudo da aplicação do Drywall em edificação vertical, Trabalho de conclusão de curso (TCC), Universidade Tecnológica Federal do Paraná.

Sandanayake, M., Zhang, G., Setunge, S., Luo, W. \& Chun-Qing, L. (2017). Estimation and comparison of environmental emissions and impacts at foundation and structure construction stages of a building: A case study. Journal of Cleaner Production, 319-329. doi:10.1016/j.jclepro.2017.03.041.

Vasques, C. P. F. \& Pisso, L. M. B. (2014). Comparativo de sistemas construtivos, convencional e wood frame em residências unifamiliares. Cognitio, 1, 1-17.

Vieira, H. (2016). Logística aplicada à construção civil como melhorar o fluxo de produção nas obras. Editora Pini.

Pfeil, W. \& Pfeil, M. (2013). Estruturas de Madeira. (6ª ed.), LTC.

Ribaski, N. G., Dudek, L. C. \& Rotta, C. E. (2017). O sistema wood frame e o setor madeireiro paranaense. Brazilian Applied Science Review, 1(1), $34-41$.

Zaccai, E. (2012). Over two decades in pursuit of sustainable development: Influence, transformations, limits. Environmental Development, 1(1), 79-90. 10.1016/j.envdev.2011.11.002

Zaparte, T. A. (2014). Estudo e adequação dos principais elementos do modelo canadense de construção em Wood Frame para o Brasil, TCC - Universidade Tecnológica Federal do Paraná - UTFPR.

Zenid, G. J. (2009). Madeira: Uso sustentável na construção civil. (2ª ed.), São Paulo, Instituto de Pesquisas Tecnológicas. 